\title{
Testing Inequality Constrained Hypotheses in SEM Models
}

\author{
Rens van de Schoot and Herbert Hoijtink \\ Department of Methods and Statistics \\ Utrecht University, The Netherlands \\ Maja Deković \\ Department of Child and Adolescent Studies \\ Utrecht University, The Netherlands
}

\begin{abstract}
Researchers often have expectations that can be expressed in the form of inequality constraints among the parameters of a structural equation model. It is currently not possible to test these so-called informative hypotheses in structural equation modeling software. We offer a solution to this problem using Mplus. The hypotheses are evaluated using plug-in $p$ values with a calibrated alpha level. The method is introduced and its utility is illustrated by means of an example.
\end{abstract}

Order-restricted inference has been studied in the frequentist framework (see, e.g., Barlow, Bartholomew, Bremner, \& Brunk, 1972; Robertson, Wright, \& Dykstra, 1988; Silvapulle \& Sen, 2004) as well as in the Bayesian framework (e.g., Hoijtink, Klugkist, \& Boelen, 2008; Klugkist, Laudy, \& Hoijtink, 2005). However, testing order constraints has received relatively little attention in the structural equation modeling (SEM) literature (Gonzalez \& Griffin, 2001; Stoel, Galindo-Garre, Dolan, \& Van den Wittenboer, 2006). SEM is often used and its attractiveness is largely due to its flexibility in specifying and testing hypotheses among both observed and latent variables in multiple groups.

SEM software can be used to impose inequality constraints among the parameters of interest. More specifically, to evaluate a research question, model parameters such as regression coefficients can be constrained to being greater or smaller than either a fixed value or other regression coefficients. We call hypotheses that contain inequality constraints informative hypotheses. Mplus (Muthén \& Muthén, 2007) allows for such user-specified constraints and order constraint parameter estimation is available. The problem is that a null hypothesis test for the evaluation of an informative hypothesis is lacking in SEM software.

Correspondence should be addressed to Rens van de Schoot, Department of Methods and Statistics, Utrecht University, P.O. Box 80.140, 3508TC, Utrecht, The Netherlands. E-mail: a.g.j.vandeschoot@uu.nl 
We offer a solution to this problem based on the parametric bootstrap method available in Mplus. Plug-in $p$ values are obtained using a likelihood ratio test. The performance of these $p$ values is evaluated and we show that the alpha level should be calibrated. Using some examples, we demonstrate how this can be done.

\section{CONSTRAINT PARAMETER ESTIMATION AND HYPOTHESIS TESTING}

Ritov and Gilula (1993) proposed to obtain maximum likelihood estimates of order-restricted models by a pooling adjacent violators algorithm (see also Robertson et al., 1988, p. 56). The procedure in Mplus (Muthén \& Muthén, 2007), the slacking parameter method, is based on the solution of Ritov and Gilula. The core of the algorithm is estimating the parameters via the maximum likelihood method such that the likelihood is maximized using the sequential quadratic programming method (Han, 1977). In this method the parameters that contain inequality constraints are updated in an iterative process where inequality constraints are treated as equality constraints whenever the estimates do not fit the constraints imposed on the parameters. This is done by the introduction of "slack" parameters into the model. See Schoenberg (1997) for more details.

How to test inequality constraint hypotheses has mainly been studied outside the SEM model. See Barlow et al. (1972), Robertson et al. (1988), and Silvapulle and Sen (2004) for a comprehensive overview. In 2002 the Journal of Statistical Planning and Inference published a special issue on testing inequality constraint hypotheses (Berger \& Ivanova, 2002; Chongcharoen, Singh, \& Wright, 2002; Khalil, Saikali, \& Berger, 2002; Lee \& Yan, 2002; Perlman \& Wu, 2002a, 2002b; Sampson \& Singh, 2002; Sen \& Silvapulle, 2002; Silvapulle, Silvapulle, \& Basawa, 2002), but none of these articles discussed constraints in SEM models. Testing informative hypotheses for SEM models has been described by Stoel et al. (2006). In this study, constraints were imposed on variance terms to obtain only positive values. Hypothesis tests were performed to test the benefit of these constraints (see also Gonzalez \& Griffin, 2001). Also, Tsonaka and Moustaki (2007) described testing parameter constraints in SEM models. They specifically described factor analysis where a parametric bootstrap was performed to obtain the results. However, they only considered a comparison between a constrained and an unconstrained model. In this study we also focus on constraint hypothesis testing within the SEM model, and although we present examples of a path model, our solution is not limited to these kind of models. We also show that the alpha values used in constraint hypothesis testing need to be calibrated, which is not done in the studies just described.

In almost all of the literature already described, the likelihood ratio test (LRT) is used to test the inequality constraint hypothesis at hand. The null distribution of this test is a chi-square distribution with degrees of freedom equal to the difference between the number of parameters of the models under comparison (Bollen, 1989). An important result from the work of Barlow et al. (1972), Robertson et al. (1988), and Silvapulle and Sen (2004) is that one of the regularity conditions of the LRT does not hold when testing inequality constraint hypotheses (see also Andrews, 1996, 2000; Ritov \& Gilula, 1993; Stoel et al., 2006). Consequently, the asymptotic distribution of the LRT is no chi-square distribution and its $p$ value cannot straightforwardly be computed. 
Moreover, model selection criteria, such as the Akaike's Information Criterion or Bayesian Information Criterion, cannot be used to distinguish between statistical models with inequality constraints between the parameters of interest. These criteria use the likelihood evaluated in its maximum as a measure of model fit, and the number of parameters of the model as a measure of complexity. The problem is that model selection criteria cannot distinguish between hypotheses when these hypotheses do not differ in model fit, but only in the number of constraints imposed on the parameters of interest.

For example, consider the hypothesis $\left\{\theta_{1}-\theta_{2}\right\}<\theta_{3}>0$ where $\theta_{1} \ldots \theta_{3}$ denote, for example, mean scores on some variable. Furthermore, suppose we want to compare this informative hypothesis to an unconstrained hypothesis where the parameters are allowed to have any value. Suppose the unconstraint parameter estimates fit the constraints, so that the estimated parameters agree with $\left\{\theta_{1}-\theta_{2}\right\}<\theta_{3}>0$. In this case, both the constraint and unconstraint hypotheses do not differ in model fit; that is, the maximum of the likelihood is the same for both models. The problem is how to account for model complexity. Because the parameters are restricted, the number of parameters used to determine model complexity is clearly not equal to 3. So far, quantifying the number of parameters for constraint hypotheses received hardly any attention in the literature.

In conclusion, evaluating informative hypothesis in SEM models is not possible with the LRT or with traditional model selection criteria. Constraint parameter estimation and informative hypothesis testing has extensively been studied, but literature for SEM models is sparse. Also, as we show in this article, calibration of the alpha level is essential when testing inequality constraint hypotheses. This received hardly any attention in the literature described before. We show how informative hypotheses can be tested in SEM models using Mplus, but we first introduce an example in which the hypotheses of interest are informative.

\section{ILLUSTRATION: ETHNICITY AND ANTISOCIAL BEHAVIOR}

The problem of testing inequality constrained hypotheses in SEM and its solution is illustrated using the following example. Deković, Wissink, and Meijer (2004) investigated whether the leading theories about antisocial behavior in the dominant culture of adolescents can be generalized to members of different ethnic groups. For this example the dominant culture is the Dutch culture, which is compared to the Moroccan, Turkish, and Surinamese cultures in the Netherlands. Three aspects of the parent-adolescent relationship were assessed: positive quality of the relationship (affection and intimacy), negative quality of the relationship (antagonism and conflict), and disclosure (how much adolescents tell the parents). The sample consists of 603 adolescents $(M$ age $=14.4$, range $=14-16$ years $) ; 68 \%$ of the adolescents are Dutch $(n=407), 11 \%$ are Moroccan $(n=68), 13 \%$ are Turkish $(n=79)$, and $8 \%$ are Surinamese $(n=49)$. Adolescents were classified into these ethnic categories according to their responses on a single item in the questionnaire: "What ethnical group best describes you?" Using these data we present three examples where the hypothesis under investigation is informative.

The structural equation model used here is given by

$$
\boldsymbol{y}_{i}^{g}=\boldsymbol{B}^{g} \boldsymbol{y}_{i}^{g}+\boldsymbol{\Gamma}^{g} \boldsymbol{x}_{i}^{g}+\zeta_{i}^{g} \quad \text { with } \boldsymbol{x}_{i} \sim N\left(\boldsymbol{\mu}_{x}^{g}, \boldsymbol{\Phi}^{g}\right)
$$


where, if $q$ is the number of dependent variables, $r$ is the number of independent variables, $g=1, \ldots, G$ denotes group membership, and $i=1, \ldots, I$ denote persons, then $\boldsymbol{y}_{i}^{g}$ is a $q \times 1$ vector of dependent variables for person $i$ within group $g, \boldsymbol{B}^{g}$ is a $q \times q$ matrix of regression coefficients between $y$ s where the diagonal must consist of zeros, $\boldsymbol{x}_{i}$ is a $r \times 1$ vector of independent variables, $\boldsymbol{\mu}_{x}^{g}$ is a $r \times 1$ vector of means for each independent variable with covariance matrix $\boldsymbol{\Phi}^{g}, \Gamma^{g}$ is $q \times r$ matrix of regression coefficients between $y \mathrm{~s}$ and $x \mathrm{~s}$, and $\zeta_{i}^{g}$ is $q \times 1$ vector with error terms that is assumed to have a multivariate-normal distribution, $\zeta_{i}^{g} \sim N\left(0, \Psi^{g}\right)$, which is independent of $\boldsymbol{y}$ and $\boldsymbol{x}$. Under these assumptions, the observed $\boldsymbol{y}_{i}$ and $\boldsymbol{x}_{i}$ have a multivariate-normal distribution with

$$
\left[\begin{array}{l}
\boldsymbol{y}_{i}^{g} \\
\boldsymbol{x}_{i}^{g}
\end{array}\right] \sim N_{q+r}\left(\begin{array}{l}
\boldsymbol{\mu}_{y}^{g} \\
\boldsymbol{\mu}_{x}^{g}
\end{array}, \Sigma^{g}\right)
$$

where $\Sigma$ represents the implied covariance matrix that is given by

$$
\Sigma^{g}=\left[\begin{array}{cc}
\Sigma_{y y}^{g} & \sum_{x y}^{g} \\
(q \times q) & (r \times q) \\
\Sigma_{y x}^{g} & \sum_{x x}^{g} \\
(q \times r) & (r \times r)
\end{array}\right]
$$

with $\mathbf{I}$ being the identity matrix and

$$
\begin{aligned}
\Sigma_{x x}^{g} & =\boldsymbol{\Phi}^{g} \\
\Sigma_{y y^{\prime}}^{g} & =\left(\mathbf{I}-\mathbf{B}^{g}\right)^{-1}\left(\boldsymbol{\Gamma}^{g} \boldsymbol{\Phi}^{g} \boldsymbol{\Gamma}^{\prime g}+\boldsymbol{\Psi}^{g}\right)\left(\mathbf{I}-\mathbf{B}^{g}\right)^{-1^{\prime}} \\
\Sigma_{x y}^{g} & =\boldsymbol{\Phi}^{g} \boldsymbol{\Gamma}^{g}\left(\mathbf{I}-\mathbf{B}^{g}\right)^{-1^{\prime}} .
\end{aligned}
$$

Let $\boldsymbol{\theta}=\left\{\boldsymbol{\theta}^{1}, \boldsymbol{\theta}^{2}\right\}$ with $\boldsymbol{\theta}^{1}=\left\{\boldsymbol{B}^{1}, \ldots, \boldsymbol{B}^{G}, \boldsymbol{\Gamma}^{1}, \ldots, \boldsymbol{\Gamma}^{G}\right\}$ and $\boldsymbol{\theta}^{2}=\left\{\boldsymbol{\Phi}^{1}, \ldots, \boldsymbol{\Phi}^{G}, \boldsymbol{\Psi}^{1}, \ldots, \boldsymbol{\Psi}^{G}\right\}$. Then, the likelihood function can be given by

$$
\log f(\boldsymbol{y}, \boldsymbol{x} \mid \boldsymbol{\theta})=\sum_{g=1}^{G}\left(\frac{\mathrm{N}^{g}}{\mathrm{~N}}\right) \mathrm{F}_{M L}^{g}\left[\mathbf{S}^{g}, \Sigma^{g}\right]
$$

where $\mathrm{N}^{g}$ is the sample size for group $g, \mathbf{S}^{g}$ is the sample covariance matrix among the observed variables in group $g, \boldsymbol{y}=\left\{\boldsymbol{y}_{1}, \ldots, \boldsymbol{y}_{N}\right\}, \boldsymbol{x}=\left\{\boldsymbol{x}_{1}, \ldots, \boldsymbol{x}_{N}\right\}$ and where $\mathrm{F}_{M L}^{g}$ is given by

$$
\mathrm{F}_{M L}^{g}=\log \left|\Sigma^{g}\right|+\operatorname{tr}\left[\mathbf{S}^{g} \Sigma^{g^{-1}}-\log \left|\mathbf{S}^{g}\right|-(\mathrm{q}+\mathrm{r})\right]
$$

We consider two types of hypothesis tests (Silvapulle \& Sen, 2004), Type A is of the form

$$
\begin{aligned}
& \mathrm{H}_{0}: \quad \mathbf{A} \boldsymbol{\theta}^{1}=\boldsymbol{c} \\
& \mathrm{H}_{1}: \quad \mathbf{A} \boldsymbol{\theta}^{1}>\boldsymbol{c},
\end{aligned}
$$

and Type $\mathrm{B}$ is of the form

$$
\begin{array}{ll}
\mathrm{H}_{0}: & \mathbf{A} \boldsymbol{\theta}^{1}>\boldsymbol{c} \\
\mathrm{H}_{1}: & \text { unconstrained, }
\end{array}
$$


where the unconstrained model refers to a model without any constraints imposed on the parameters and where, if $m$ is the number of inequality constraints imposed on the model and $k$ the number of parameters involved, $\mathbf{A}$ is an $m \times k$ matrix of known constants, and $\mathbf{c}$ is an $m \times 1$ vector of known constants. More specific examples will be given in the sequel.

\section{Example 1: Simple Regression}

The first example is a simple regression model where levels of antisocial behavior are regressed on either a negative or positive relation with the parent and adolescent disclosure (see Figure 1), where $\boldsymbol{B}=0$ and

$$
\begin{aligned}
& \boldsymbol{\Gamma}=\left[\begin{array}{lll}
\gamma_{1} & \gamma_{2} & \gamma_{3}
\end{array}\right], \\
& \Psi=\left[\psi_{1}\right], \\
& \Phi=\left[\begin{array}{lll}
\phi_{11} & & \\
\phi_{12} & \phi_{22} & \\
\phi_{13} & \phi_{23} & \phi_{33}
\end{array}\right]
\end{aligned}
$$

Note that this model is based on the total sample, therefore the superscript $g$ is not needed.

Deković et al. (2004) stated that adolescent disclosure is the strongest predictor of antisocial behavior, followed by either a negative or positive relation with the parent (see also Dishion $\&$ McMahon, 1998). We therefore hypothesize that the regression coefficients $\gamma_{1}$ and $\gamma_{2}$ are smaller than $\gamma_{3}$. Using

$$
\begin{gathered}
\boldsymbol{A}=\left[\begin{array}{ccc}
-1 & 0 & 1 \\
0 & -1 & 1
\end{array}\right], \\
\boldsymbol{\theta}^{1}=\left[\begin{array}{l}
\gamma_{1} \\
\gamma_{2} \\
\gamma_{3}
\end{array}\right], \\
\boldsymbol{c}=\left[\begin{array}{l}
0 \\
0
\end{array}\right],
\end{gathered}
$$

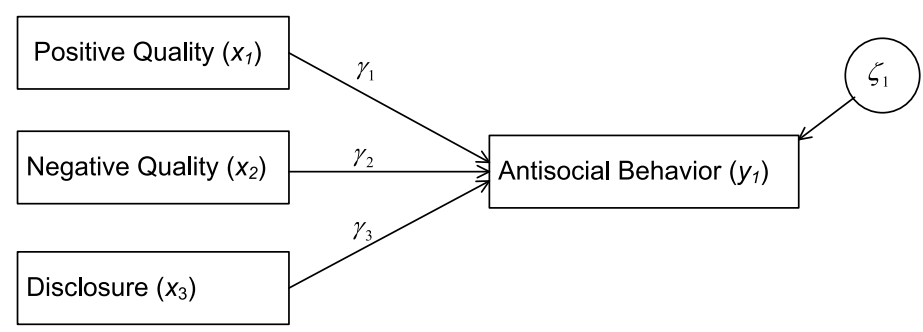

FIGURE 1 Path model among relationship characteristics, disclosure, and prevalence of antisocial behavior. 
the hypotheses tested for this example with $m=2$, are for Type A

$$
\begin{array}{ll}
H_{0}: & {\left[\begin{array}{l}
\gamma_{3}-\gamma_{1}=0 \\
\gamma_{3}-\gamma_{2}=0
\end{array}\right]} \\
\text { versus } & \\
H_{1}: & {\left[\begin{array}{l}
\gamma_{3}-\gamma_{1}>0 \\
\gamma_{3}-\gamma_{2}>0
\end{array}\right],}
\end{array}
$$

and for Type B

$$
H_{0}: \quad\left[\begin{array}{l}
\gamma_{3}-\gamma_{1}>0 \\
\gamma_{3}-\gamma_{2}>0
\end{array}\right]
$$

versus

$$
H_{1}: \quad\left[\begin{array}{ll}
\gamma_{3}, & \gamma_{1} \\
\gamma_{3}, & \gamma_{2}
\end{array}\right] \text {. }
$$

Note that $H_{1}$ in Equation 16 can also be written as $\left\{\gamma_{1}, \gamma_{2}\right\}<\gamma_{3}$. This type of notation will be used in the remainder of this article. We used Mplus version 5 (Muthén \& Muthén, 2007) to estimate the unconstrained and constrained regression coefficients (see Table 1). As can be seen in this table, the unconstrained estimate of $\gamma_{2}$ is not smaller than $\gamma_{3}$. Consequently, the constrained estimates of $\gamma_{2}$ and $\gamma_{3}$ are set equal by the introduction of a "slack" parameter (see the lower panel of Table 1).

\section{Example 2: Multigroup Analysis}

Research about the nature and impact of antisocial behavior is dominated by studies conducted with White, Western, middle-class adolescents (Deković et al., 2004). It could be questioned whether the model in Figure 1 is the same for different ethnic groups living in The Netherlands: Dutch (indicated by $g=1)$, Turkish $(g=2)$, Moroccan $(g=3)$, and Surinamese adolescents

TABLE 1

Regression Coefficients for Example 1

\begin{tabular}{lll}
\hline Coefficient & $B$ & $S E$ \\
\hline Unconstrained & & \\
$\gamma_{1}$ & .06 & .02 \\
$\gamma_{2}$ & .24 & .03 \\
$\gamma_{3}$ & .23 & .03 \\
Constrained & & \\
$\gamma_{1}$ & .06 & .02 \\
$\gamma_{2}$ & .235 & .03 \\
$\gamma_{3}$ & .235 & .03 \\
\hline
\end{tabular}


$(g=4)$, where $\boldsymbol{B}=0$ and

$$
\begin{gathered}
\Gamma^{1}=\left[\begin{array}{lll}
\gamma_{1}^{1} & \gamma_{2}^{1} & \gamma_{3}^{1}
\end{array}\right] \\
\vdots \\
\Gamma^{4}=\left[\begin{array}{lll}
\gamma_{1}^{4} & \gamma_{2}^{4} & \gamma_{3}^{4}
\end{array}\right], \\
\Psi^{1}=\left[\begin{array}{ll}
\psi_{1}^{1}
\end{array}\right] \\
\vdots \\
\Psi^{4}=\left[\psi_{1}^{4}\right], \\
\Phi^{1}=\left[\begin{array}{lll}
\phi_{11}^{1} & \\
\phi_{12}^{1} & \phi_{22}^{1} \\
\phi_{13}^{1} & \phi_{23}^{1} & \phi_{33}^{1}
\end{array}\right] \\
\Phi^{4}=\left[\begin{array}{lll}
\phi_{11}^{4} & & \\
\phi_{12}^{4} & \phi_{22}^{4} & \\
\phi_{13}^{4} & \phi_{23}^{4} & \phi_{33}^{4}
\end{array}\right] .
\end{gathered}
$$

The null hypothesis is that the regression coefficients for the predictors of antisocial behavior are the same for all ethnic groups (see, e.g., Greenberger \& Chen, 1996):

$$
H_{0}: \quad\left[\begin{array}{l}
\gamma_{1}^{1}=\gamma_{1}^{2}=\gamma_{1}^{3}=\gamma_{1}^{4} \\
\gamma_{2}^{1}=\gamma_{2}^{2}=\gamma_{2}^{3}=\gamma_{2}^{4} \\
\gamma_{3}^{1}=\gamma_{3}^{2}=\gamma_{3}^{3}=\gamma_{3}^{4}
\end{array}\right]
$$

According to Deković et al. (2004) there are also indications in the literature that the same risk factors have different effects in different ethnic groups, a so-called process times context interaction phenomenon. The authors expected that cross-ethnic variations result in weaker relations between parent-child relations and adolescent behavior compared to Dutch families. This was mainly expected because of differences in family expectations (Phalet \& Schönpflung, 2001) and differences in intergenerational conflicts due to migration (Deković, Noom, \& Meeus, 1997). The informative hypothesis $H_{1}$ is:

$$
H_{1}: \quad\left[\begin{array}{l}
\gamma_{1}^{1}>\left\{\gamma_{1}^{2}, \gamma_{1}^{3}, \gamma_{1}^{4}\right\} \\
\gamma_{2}^{1}>\left\{\gamma_{2}^{2}, \gamma_{2}^{3}, \gamma_{2}^{4}\right\} \\
\gamma_{3}^{1}>\left\{\gamma_{3}^{2}, \gamma_{3}^{3}, \gamma_{3}^{4}\right\}
\end{array}\right] \text {. }
$$

The hypotheses that are tested for this example are for Type A: Equation 20 versus Equation 21; and for Type B: Equation 21 versus the unconstrained model. In Table 2 the unconstrained and constrained regression coefficients are shown. As can be seen, not all unconstraint regression coefficients are in agreement with the constraints of $H_{1}$ in Equation 21. For example, $\gamma_{2}$ for the Dutch adolescents is smaller instead of higher than $\gamma_{2}$ for Moroccan adolescents. The bottom of Table 2 renders parameter estimates, obtained with the parameter slack method, that are in agreement with the constraints. 
TABLE 2

Regression Coefficients for Dutch, Moroccan, Turkish, and Surinamese

Adolescents for Example 2

\begin{tabular}{lllll}
\hline & \multicolumn{4}{c}{ Ethnicity } \\
\cline { 2 - 5 } Coefficient & Dutch & Moroccan & Turkish & Surinamese \\
\hline Unconstrained & & & & \\
$\gamma_{1}$ & $.05(.03)$ & $.12(.09)$ & $.04(.08)$ & $.15(.09)$ \\
$\gamma_{2}$ & $.28(.03)$ & $.23(.11)$ & $.16(.08)$ & $.08(.11)$ \\
$\gamma_{3}$ & $.20(.04)$ & $.33(.13)$ & $.25(.10)$ & $.24(.14)$ \\
Constrained & & & & $.03(.07)$ \\
$\gamma_{1}$ & $.06(.02)$ & $.06(.02)$ & $.03(.02)$ \\
$\gamma_{2}$ & $.28(.03)$ & $.28(.03)$ & $.16(.08)$ & $.08(.11)$ \\
$\gamma_{3}$ & $.22(.03)$ & $.22(.03)$ & $.22(.03)$ & $.16(.12)$ \\
\hline
\end{tabular}

Note. Values in parentheses represent standard error.

\section{Example 3: Path Model}

The third example includes the variable hanging around with deviant peers. The hypothesis states that problem behavior is not only directly predicted by disclosure and a negative or positive relation with the parent, but is also indirectly predicted via hanging around with deviant peers (see Figure 2), where

$$
\begin{gathered}
\boldsymbol{B}=\left[\begin{array}{cc}
0 & 0 \\
\beta_{21} & 0
\end{array}\right], \\
\boldsymbol{\Gamma}=\left[\begin{array}{lll}
\gamma_{11} & \gamma_{12} & \gamma_{13} \\
\gamma_{21} & \gamma_{22} & \gamma_{23}
\end{array}\right], \\
\Psi=\left[\begin{array}{cc}
\psi_{11} & \\
0 & \psi_{22}
\end{array}\right], \\
\Phi=\left[\begin{array}{ccc}
\phi_{11} & \\
\phi_{12} & \phi_{22} & \\
\phi_{13} & \phi_{23} & \phi_{33}
\end{array}\right] .
\end{gathered}
$$

Note that this model is based on the total sample, therefore superscript $g$ is not needed.

As argued by Deković et al. (2004), children spend, especially in adolescence, more and more time with their peers without adult supervision (see also Mounts \& Steinberg, 1995). During this period peers become the most important reference group for adolescents. Deković et al. (2004) stated that especially in this period the association with deviant peers has emerged as the most prominent predictor of problem behavior. The hypotheses tested for Example 3 are hypothesis Type A:

$$
\begin{array}{ll}
H_{0}: & \beta_{21}=\gamma_{21}=\gamma_{22}=\gamma_{23} \\
\text { versus } & \\
H_{1}: & \beta_{21}>\left\{\gamma_{21}, \gamma_{22}, \gamma_{23}\right\}
\end{array}
$$




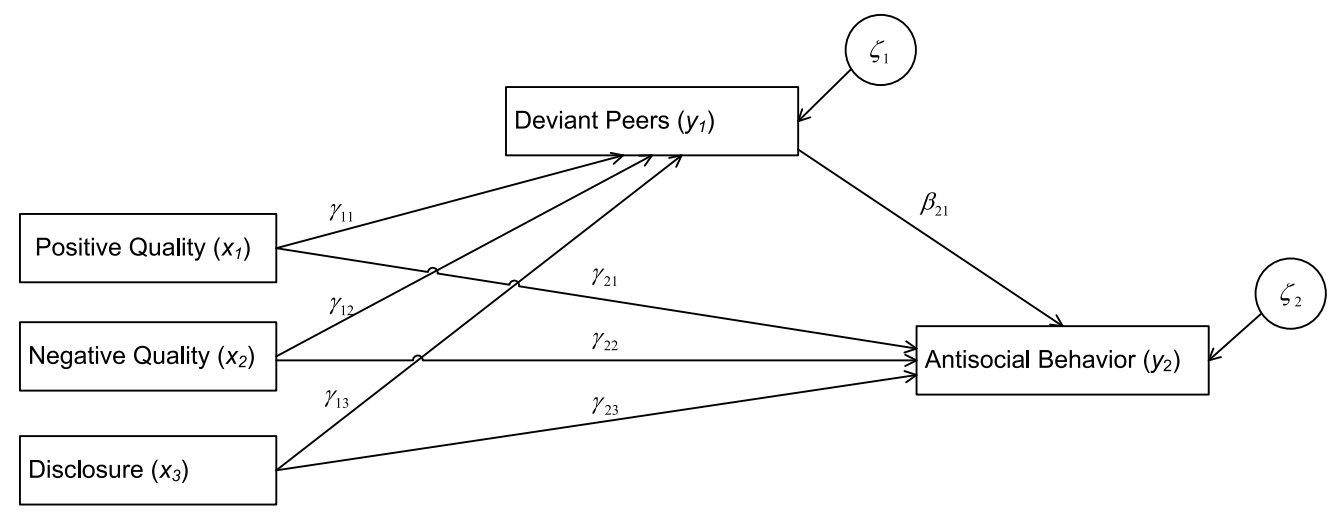

FIGURE 2 Path model among relationship characteristics, hanging around with deviant peers, and prevalence of antisocial behavior.

and hypothesis Type B

$$
\begin{array}{ll}
H_{0}: & \beta_{21}>\left\{\gamma_{21}, \gamma_{22}, \gamma_{23}\right\} \\
\text { versus } & \\
H_{1}: & \beta_{21}, \gamma_{21}, \gamma_{22}, \gamma_{23} .
\end{array}
$$

The unconstrained and constrained regression coefficients are shown in Table 3. As can be seen in Table 3, the constrained coefficients do not differ from their unconstrained counterparts. Hence, the constraints imposed by the informative hypothesis are not contradicted by the data.

\section{PARAMETRIC BOOTSTRAP}

To evaluate informative hypotheses like those presented in the previous section we make use of the parametric bootstrap. Bootstrapping is an approach for statistical inference falling within a broader class of resampling methods (Efron \& Tibshirani, 1993). Various authors have

TABLE 3

Regression Coefficients for Example 3

\begin{tabular}{lll}
\hline Coefficient & $B$ & $S E$ \\
\hline Unconstrained & & \\
$\gamma_{11}$ & .05 & .03 \\
$\gamma_{12}$ & .31 & .03 \\
$\gamma_{13}$ & .22 & .04 \\
$\beta_{21}$ & .55 & .02 \\
Constrained & & .03 \\
$\gamma_{11}$ & .05 & .03 \\
$\gamma_{12}$ & .31 & .04 \\
$\gamma_{13}$ & .22 & .02 \\
$\beta_{21}$ & .55 & \\
\hline
\end{tabular}


suggested using the parametric bootstrap when the parameter space is restricted (Galindo-Garre \& Vermunt, 2004, 2005; Ritov \& Gilula, 1993; Stoel et al., 2006; Tsonaka \& Moustaki, 2007).

\section{Bootstrap Method}

The method we advocate starts with the observed data $z=\{\boldsymbol{y}, \boldsymbol{x}\}$ and the likelihood in Equation 5 (see Start in Figure 3). Step 1 is a parametric bootstrap from a population in which the null hypothesis is true. First, $\theta$ is estimated under $H_{0}$ using the data $z$ resulting in

$$
f\left(z \mid \hat{\theta}_{H_{0} \mid z}\right)
$$

Using Equation 28, $T$ bootstrap samples of size $n$ are generated, resulting in data sets $z_{t}^{r e p}$, for $t=1, \ldots, T$ (see Figure 3). Then, $\theta$ is estimated for each replicated data set under $H_{0}$, rendering

$$
f\left(z_{1}^{r e p} \mid \hat{\theta}_{H_{0} \mid z_{1}^{r e p}}\right) \ldots f\left(z_{T}^{r e p} \mid \hat{\theta}_{H_{0} \mid z_{T}^{r e p}}\right)
$$

Further, $\theta$ is estimated under $H_{1}$, rendering

$$
f\left(z_{1}^{r e p} \mid \hat{\theta}_{H_{1} \mid z_{1}^{r e p}}\right) \ldots f\left(z_{T}^{r e p} \mid \hat{\theta}_{H_{1} \mid z_{T}^{r e p}}\right)
$$

The second step, denoted by Step 2 in Figure 3, is to repeat these computations conditional on the observed data set and to compute $f\left(z \mid \hat{\theta}_{H_{0} \mid z}\right)$ and $f\left(z \mid \hat{\theta}_{H_{1} \mid z}\right)$.

The final step (see the lower part of the top panel of Figure 3 ) is to choose a test statistic, denoted by $\Lambda$, to investigate the compatibility of the null hypothesis with the observed data. Like many previous studies (e.g., Barlow et al., 1972; Robertson et al., 1988, Silvapulle \& Sen, 2004), we also use the LRT for evaluating the hypotheses at hand, but, as illustrated before, we do not use a $p$ value based on a chi-square distribution.

Because $f(z \mid \theta)$ is proportional to the likelihood, an LRT is performed for each replicated data set rendering

$$
\Lambda_{t}=-2 \log \left\{\frac{f\left(z_{t}^{r e p} \mid \hat{\theta}_{\left.H_{0} \mid z_{t}^{r e p}\right)}\right.}{f\left(z_{t}^{r e p} \mid \hat{\theta}_{H_{1} \mid z_{t}^{r e p}}\right)}\right\}
$$

and for the observed data set it renders

$$
\Lambda=-2 \log \left\{\frac{f\left(z \mid \hat{\theta}_{H_{0} \mid z}\right)}{f\left(z \mid \hat{\theta}_{H_{1} \mid z}\right)}\right\} .
$$

Now, a $p$ value can be computed using

$$
p=\mathrm{P}\left(\Lambda_{t}>\Lambda \mid H_{0}, z\right)
$$




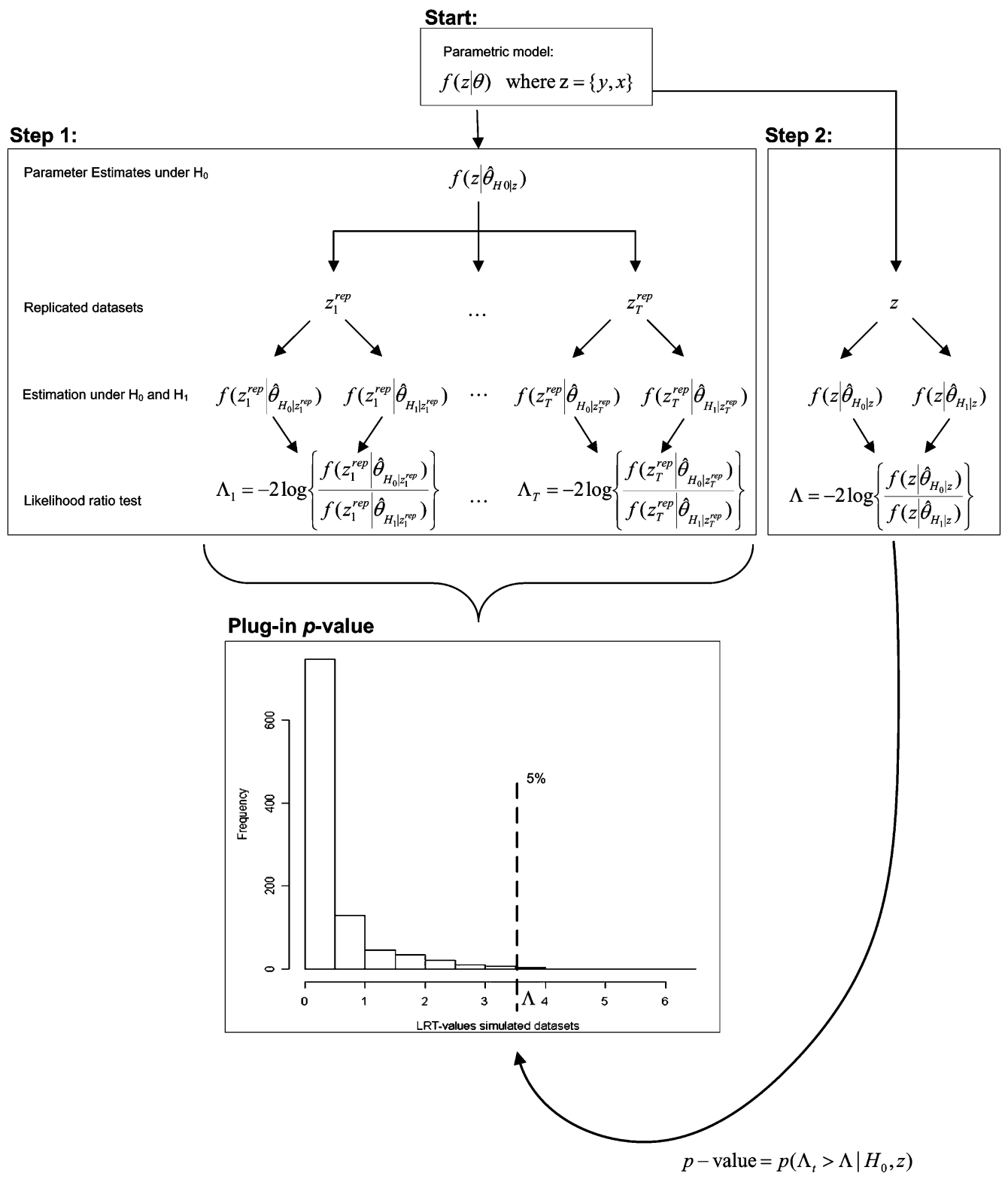

FIGURE 3 Graphical representation of the parametric bootstrap method. 
It can be approximated by the proportion of LRT values from the simulated data sets that are equal or larger than the LRT value of the observed data set, resulting in the definition of the plug-in $p$ value

$$
p \approx \frac{\sum_{t=1}^{\mathrm{T}} I_{t}}{\mathrm{~T}},
$$

where $I_{t}$ is an indicator function taking the value 1 if the inequality holds and 0 otherwise:

$$
I_{t}= \begin{cases}1 & \text { if } \Lambda_{t}>\Lambda \\ 0 & \text { otherwise }\end{cases}
$$

A hypothetical distribution is illustrated in the graph in the lower part of Figure 3. To determine whether the $\Lambda$ value from the observed data set stems from a population where the null hypothesis is true, it has to be smaller than a chosen alpha value. Traditionally an alpha value of .05 is used and as such the observed $\Lambda$ value has to lie on the left side of the dotted line in Figure 3. However, as we will show in the next section, in many situations the alpha value needs to be calibrated.

The procedure previously described can be conducted for Type A and B hypothesis testing. The parameter estimates and likelihood values can be obtained using Mplus and we developed $\mathrm{R}$ code that automatically computes the LRT values and the plug-in $p$ value from the output files of Mplus. Input files for all examples and $\mathrm{R}$ code can be downloaded from http://www. fss.uu.nl/ms/schoot.

\section{FREQUENCY PROPERTIES OF THE ASYMPTOTIC $p$ VALUES}

In the previous section we showed how to obtain plug-in $p$ values for the evaluation of informative hypotheses. An appealing property for any $p$ value, and consequently for our plug-in $p$ value, is, considered as a random variable, to be asymptotically uniform $[0,1]$ under the null hypothesis: $\mathrm{P}\left(p<\alpha \mid H_{0}\right)=\alpha$. However, in some situations exact uniformity of $p$ values cannot be attained (Andrews, 2000; Bayarri \& Berger, 2000; Galindo-Garre \& Vermunt, 2004, 2005; Stoel et al., 2006). Andrews (2000), for example, showed that the results of the bootstrap procedure are not coherent when inequality constraints are imposed on the model parameters. Furthermore, Galindo-Garre and Vermunt (2004) showed in a simulation study that the parametric bootstrap can produce $p$ values that are higher than expected.

The bootstrap procedure described in the previous section can also lead to $p$ values that are biased. To determine whether the actual alpha level differs from its nominal level a double bootstrap procedure is used (Efron \& Tibshirani, 1993). This procedure renders a calibrated alpha level: $\mathrm{P}\left(p<\alpha^{*} \mid H_{0}\right)=\alpha$, where $\alpha *$ denotes the calibrated alpha level.

As can be seen in Figure 4, in the double bootstrap procedure there are two stages of bootstrapping. In Stage 1, data sets are generated using $f\left(z \mid \hat{\theta}_{H_{0} \mid z}\right)$. Note that we make an important assumption here. We implicitly assume that in our procedure $\hat{\theta}_{H_{0} \mid z}$ is a good approximation of the true population values $\theta_{H_{0}}$, which are unknown. We assume that with $n$ sufficiently large, the true population values will be close to the estimated values $f\left(z \mid \hat{\theta}_{H_{0} \mid z}\right)$. 


\section{Stage 1:}

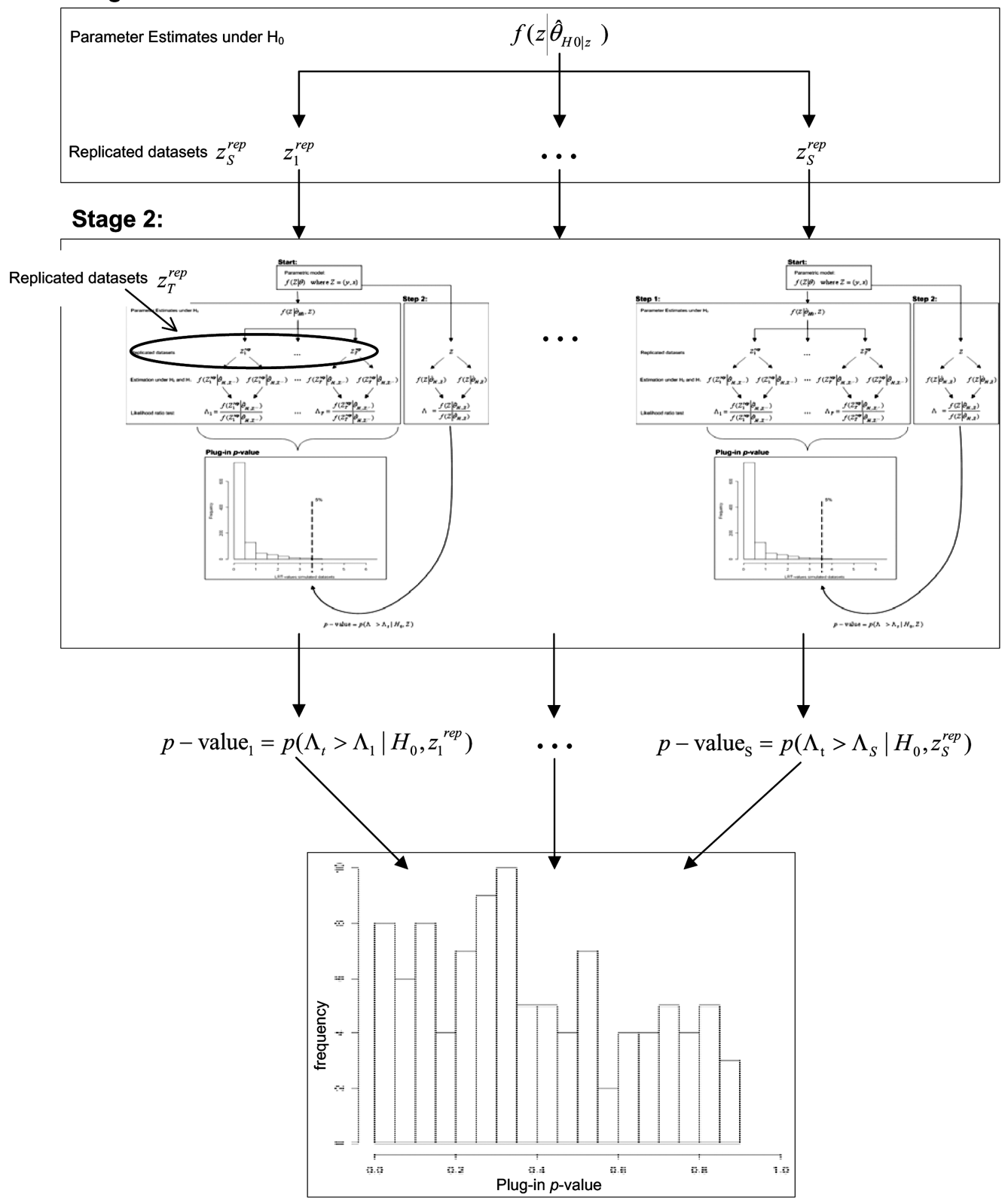

FIGURE 4 Graphical representation of the double bootstrap method. 
The result of Stage 1, are $S$ data sets (for $s=1, \ldots, S$ ) and the double bootstrap algorithm amounts to treating each bootstrap sample $z_{s}^{\text {rep }}$ like an original data set in the second stage of the double bootstrap procedure (see Stage 2 in Figure 4). For each first-stage data set, $z_{s}^{r e p}$, a plug-in $p$ value can be computed based on the procedure described in the previous section.

In total, $S$ plug-in $p$ values are computed and a hypothetical distribution of these values is shown in the lower part of Figure 4. As can be seen, it does not have a uniform distribution, but is skewed. In Figure 4, the 5th percentile of generated plug-in $p$ values has a plug-in $p$ value of .02. That is, $5 \%$ of the $p$ values are smaller than .02 , and for example $11 \%$ of the $p$ values are smaller than .05 . In such a case, the alpha level for evaluation of the $p$ value computed for the observed data set needs to be calibrated. That is, the $p$ value should be compared to $\alpha^{*}=.02$ instead of $\alpha=.05$, because $\mathrm{P}\left(p<.02 \mid H_{0}\right)=.05$.

\section{RESULTS FOR EXAMPLES}

\section{Example 1}

To evaluate the performance of the plug-in $p$ value for Example 1, in total four double bootstraps are performed with $S=1,000$ and $T=1,000$ : (a) hypothesis test Type A with $n=50$; (b) hypothesis test Type B with $n=50$; (c) hypothesis test Type A with $n=640$; and (d) hypothesis test Type B with $n=640$. In Figure 5 the four corresponding distributions of plug-in $p$ values are displayed.

As can be seen in Figure 5a and $5 \mathrm{c}$ the distribution for hypothesis test Type A is almost uniform for both $n=50$ and $n=640$ with $\mathrm{P}\left(p<.048 \mid H_{0}\right)=.05$ and $\mathrm{P}\left(p<.046 \mid H_{0}\right)=.05$, respectively. As was shown by Silvapulle \& Sen (2004, pp. 32-33), the $p$ values of this statistical model and for hypothesis test Type A are uniformly distributed. Our results for small and large sample sizes are pretty close to being uniform and the small deviations are sampling errors. Hence, the traditional alpha level of $\alpha=.05$ is used to evaluate the results for the observed data set.

For hypothesis test Type B, however, the distribution is clearly not uniform, see the distributions in Figure 5b and 5d. High values of the plug-in $p$ value do not exist and low values appear too often. For small $n, \mathrm{P}\left(p<.02 \mid H_{0}\right)=.05$ (see Table 4) and the alpha level for the analysis with the observed data set needs to be calibrated, $\alpha^{*}=.02$. Accidentally, for large $n, \mathrm{P}\left(p<.048 \mid H_{0}\right)=.05$ and $\alpha^{*}$ does not need to be calibrated much, $\alpha^{*}=.048$. However, as can be seen in Figure $5 \mathrm{~d}$ the distribution is clearly not uniform, for example, $\mathrm{P}\left(p<.30 \mid H_{0}\right)=.50$ and $\mathrm{P}\left(p<.38 \mid H_{0}\right)=.70$, indicating that calibration is necessary for different alpha levels.

To evaluate the hypotheses in Equations 15 and 16 for the observed data set, a parametric bootstrap is performed where 1,000 data sets were generated. Each of these samples was fitted under $H_{0}$ for hypothesis test Type $\mathrm{A}$ and $\mathrm{B}$. Based on the results shown in Table 4, it can be concluded that for hypothesis test Type A, $H_{0}$ can be rejected $(p<.001, \alpha=.05)$. This implies that the hypothesis $H_{0}: \gamma_{1}=\gamma_{2}=\gamma_{3}$ is rejected in favor of the informative hypothesis, $H_{1}:\left\{\gamma_{1}, \gamma_{2}\right\}<\gamma_{3}$. Moreover, the result of hypothesis test Type B indicates that the informative hypothesis is rejected $\left(p<.001, \alpha^{*}=.048\right)$ in favor of the unconstrained 


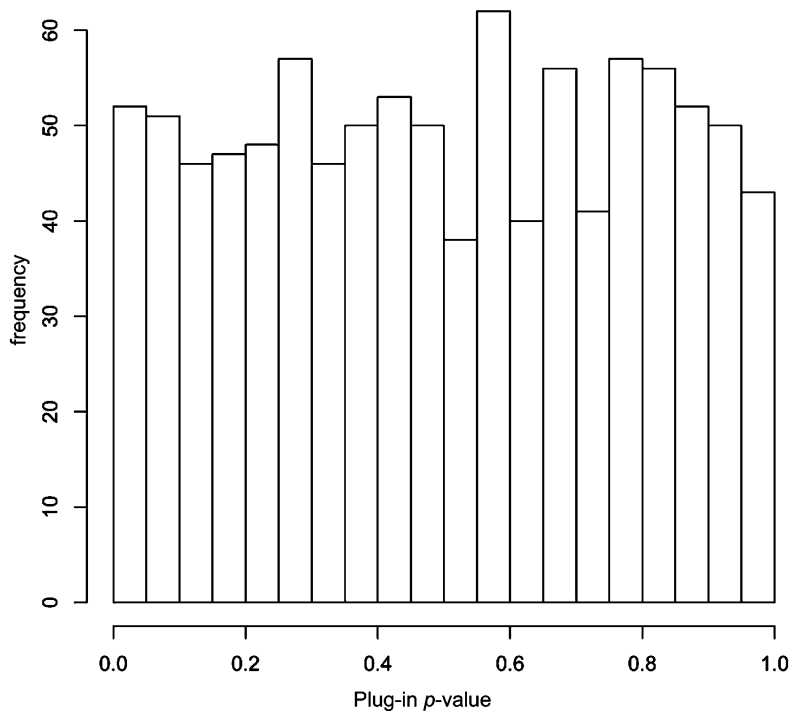

(a)

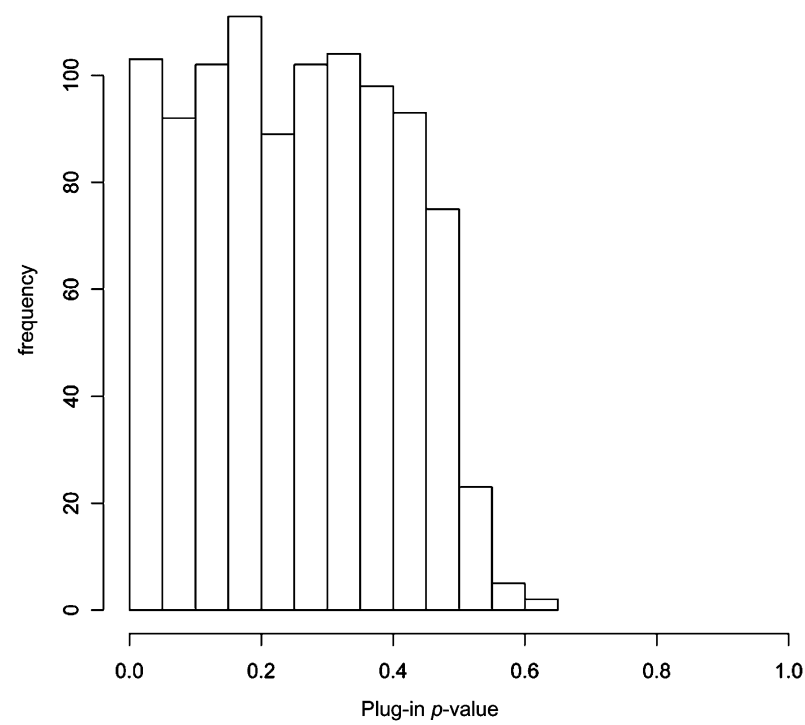

(b)

FIGURE 5 Distribution of plug-in $p$ values for Example 1: In (a) and (c), hypothesis test Type A is evaluated; in (b) and (d) hypothesis test Type B is evaluated; in (a) and (b) $n=50$; in (c) and (d) $n=640$. (continued) 


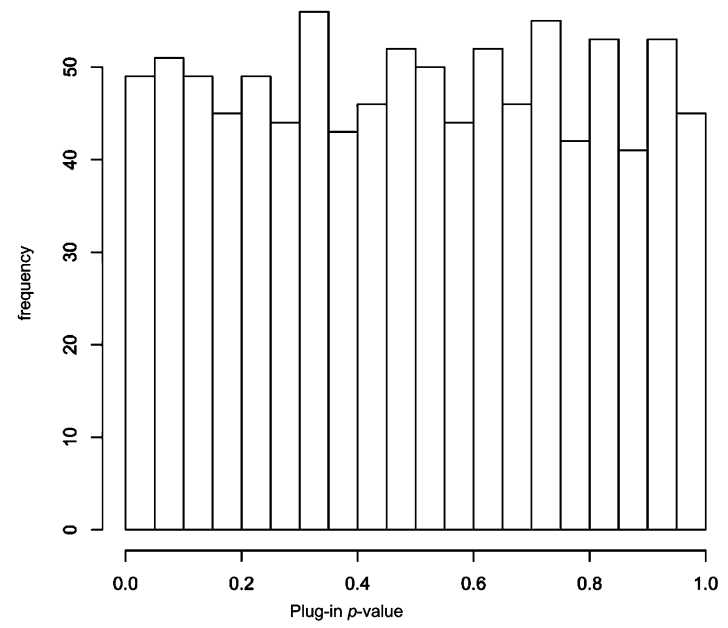

(c)

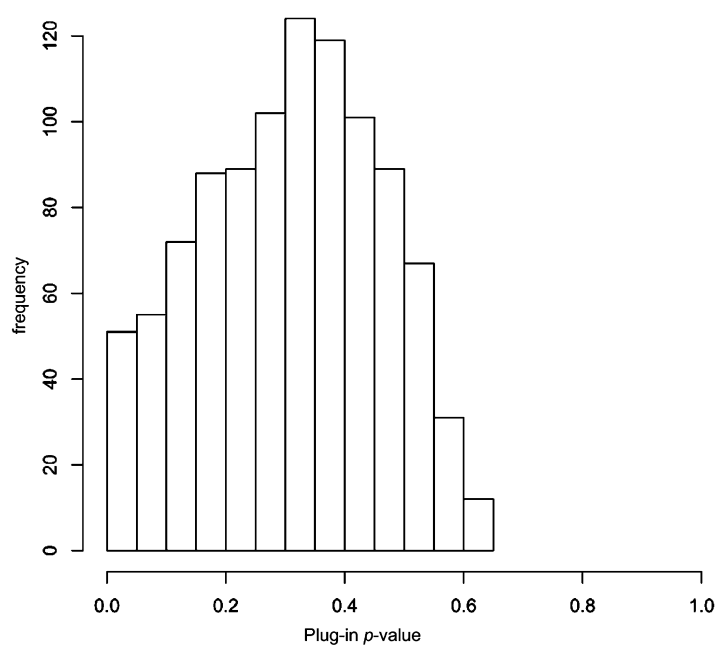

(d)

FIGURE 5 (Continued).

model $H_{1}: \gamma_{1}, \gamma_{2}, \gamma_{3}$. Inspection of Table 1 reveals that $\gamma_{2}>\gamma_{3}$ and as such the unconstrained parameter estimates do not fit either $H_{0}: \gamma_{1}=\gamma_{2}=\gamma_{3}$ or $H_{1}:\left\{\gamma_{1}, \gamma_{2}\right\}<\gamma_{3}$.

In conclusion, levels of antisocial behavior are not evenly predicted by how much adolescents tell the parents and by either a positive or a negative quality of the relationship with the parents (rejection of $H_{0}$ ). However, the expectation that disclosure is the best predictor does not hold (rejection of $H_{1}$ ). 
TABLE 4

Results for the Double Bootstrap Procedure and the Parametric Bootstrap Procedure for Examples 1 to 3

\begin{tabular}{lllcc}
\hline & Hypothesis Test & $\alpha^{*}$ & $\Lambda$ & Plug-In p Value \\
\hline Example 1 & Type A $(n=50)$ & .048 & - & - \\
& Type A $(n=640)$ & .046 & 42.01 & $<.001$ \\
& Type B $(n=50)$ & .024 & - & - \\
Example 2 & Type B $(n=640)$ & .048 & 17.41 & $<.001$ \\
& Type A & .038 & 2.55 & .49 \\
Example 3 & Type B & .058 & 1.07 & .46 \\
& Type A & .056 & 132.42 & $<.001$ \\
& Type B & - & 0.0 & $>.999$ \\
\hline
\end{tabular}

\section{Example 2}

To evaluate the hypotheses for Example 2, two double bootstraps are performed to determine the correct alpha level for hypothesis test Type A and Type B, with $S=500, T=500$, and group sizes for group $g=1, \ldots, 4$ equal to the sample sizes.

The results are shown in Table 4 and the distribution of $p$ values is shown in Figure 6a and $\mathrm{b}$ for hypothesis test Type $\mathrm{A}$ and $\mathrm{B}$, respectively. The hypothesis $H_{0}$, shown in Equation 20 , cannot be rejected in favor of hypothesis $H_{1}\left(p=.49, \alpha^{*}=.04\right)$, shown in Equation 21. For hypothesis test Type B the informative hypothesis cannot be rejected in favor of the unconstrained hypothesis $\left(p=.46, \alpha^{*}=.056\right)$. So, after testing the informative hypothesis it appears that the observed differences shown in Table 2 are too small to reject $H_{0}$ shown in Equation 20. This makes sense because confidence intervals, if computed row-wise using 1.96 times $S E$, overlap and as such provide a lot of support for $H_{0}$ in Equation 20.

In conclusion, compared to Dutch adolescents, adolescents from different ethnic groups are satisfied to a similar degree with their relationships with parents. Besides, Dutch adolescents disclose as much information as adolescents from different ethnic groups.

\section{Example 3}

For Example 3, a double bootstrap was performed with $S=1,000, T=1,000$ and $n=640$ for hypothesis test Type A and B. The results are shown in Table 4 and the distribution of $p$ values for hypothesis test Type $\mathrm{A}$ is shown in Figure 7. For this hypothesis test, the null hypotheses is rejected in favor of the informative hypothesis $\left(p<.001, \alpha^{*}=.04\right)$.

For hypothesis test Type B, it appears that for all $S$ bootstraps $p=1$. This result implies that in none of the $S$ bootstraps did the constraint parameter estimates violate the inequality constraints imposed on the regression coefficients. Also, for the observed data set, $p=1$. We wanted $\alpha$ to have the property $\mathrm{P}\left(p<.05 \mid H_{0}\right)=.05$, but for this example we observed $\mathrm{P}\left(p<.05 \mid H_{0}\right)=0$. This simulation study shows that that we cannot make an incorrect conclusion with respect to hypothesis test Type B.

Thus, the association with deviant peers is the most prominent predictor of problem behavior. A visual inspection of Table 3 confirms this conclusion because the unconstraint estimate $\beta_{21}$ is larger than the unconstraint estimates of $\gamma_{21}, \gamma_{22}$, and $\gamma_{23}$. 


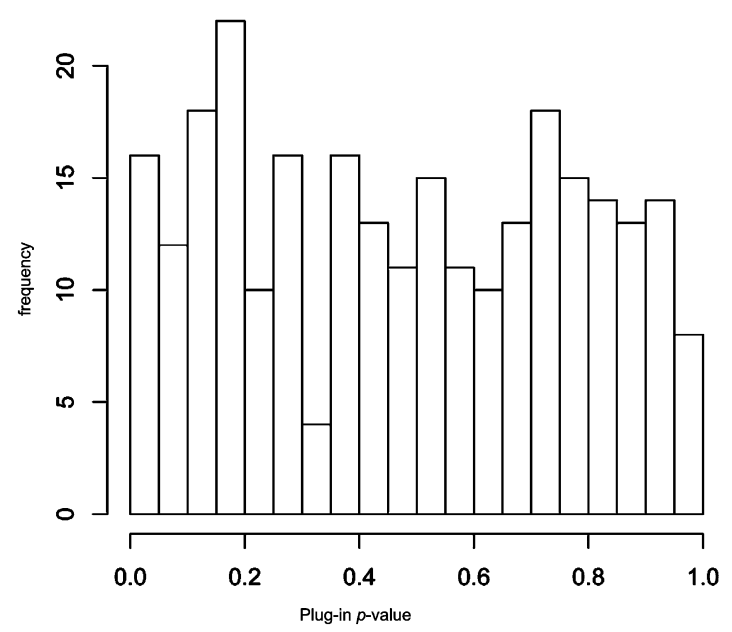

(a)

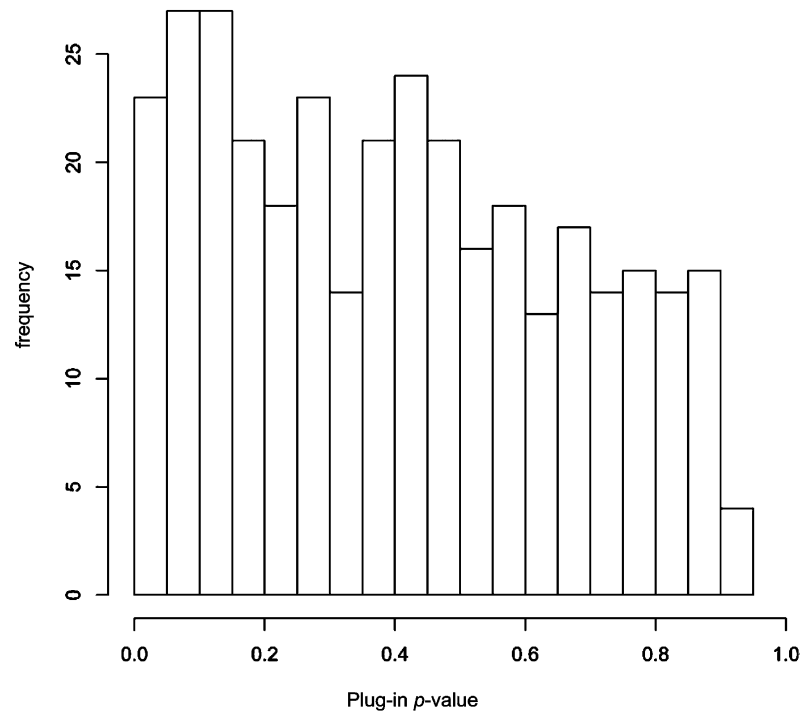

(b)

FIGURE 6 Distribution of plug-in $p$ values for Example 2: In (a) test Type A is evaluated, and in (b) test Type $B$ is evaluated. 


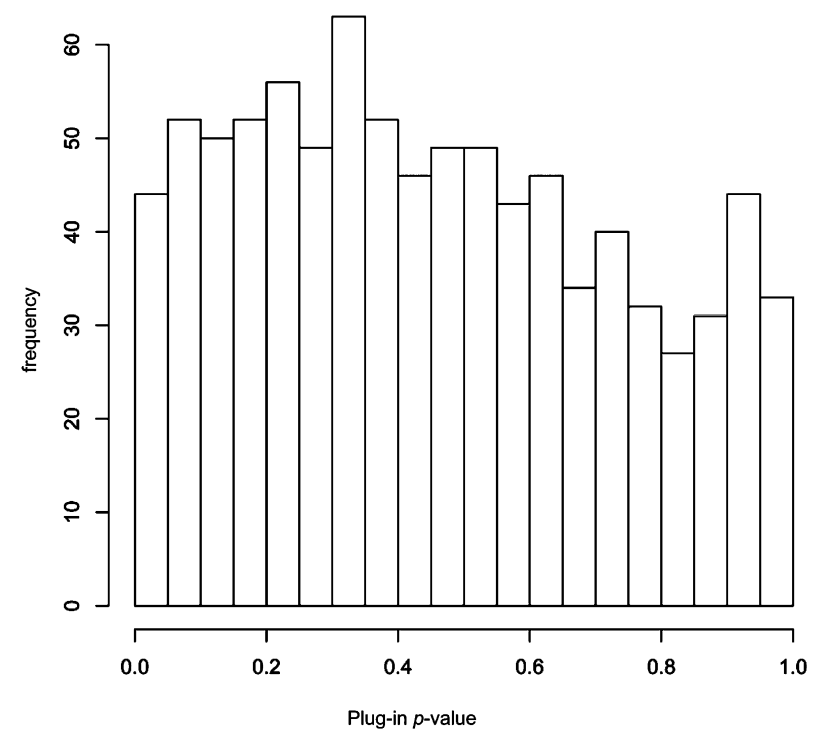

FIGURE 7 Distribution of plug-in $p$ values for Example 3, test Type A.

\section{CONCLUSION}

Traditional hypotheses tests and model selection criteria are not equipped to deal with informative hypotheses formulated in terms of inequality constraints among the parameters of a structural equation model. In this article we presented a solution for this problem using Mplus. Some issues that need further elaboration are now discussed.

First, $p$ values are often used in SEM and are evaluated using the traditional alpha level of .05 . Using the double bootstrap procedure we evaluated the frequency properties of the plug-in $p$ values resulting from our method. These results show clearly that the distribution of the $p$ values is not always uniform and calibration is needed. This is especially the case when evaluating hypotheses of Type B.

Second, we used rather simple SEM models. However, there are no technical limitations to using inequality constraints for more complicated models in Mplus, for example, including latent effects, second-order effects, or categorical variables. Figure 8 shows a hypothetical SEM model with one latent variable and seven observed variables. For this model constraints could be imposed on, for example, $\gamma_{1} \ldots \gamma_{3}$. In this article we only discussed informative hypotheses with inequality constraints of the type $\gamma_{1}>\gamma_{2}>\gamma_{3}$. There are, however, no technical limitations to evaluate an informative hypothesis consisting of combinations of equality and inequality constraints of the form $\gamma_{1}>\gamma_{2}=\gamma_{3},\left\{\gamma_{1}-\gamma_{2}\right\}>2$, or $\left\{\gamma_{1}-\gamma_{2}\right\}<\gamma_{3}>1$.

A limitation of the procedure is that computational time can be substantial. To compute the examples in this article we used Pentium computers $(3.20 \mathrm{MHz})$ containing two dual processors (Mplus can deal with multiple processors) with 1 GB memory. The models for Example 1 and 3 took approximately 2 days to compute, but Example 2 took more than 2 weeks. 


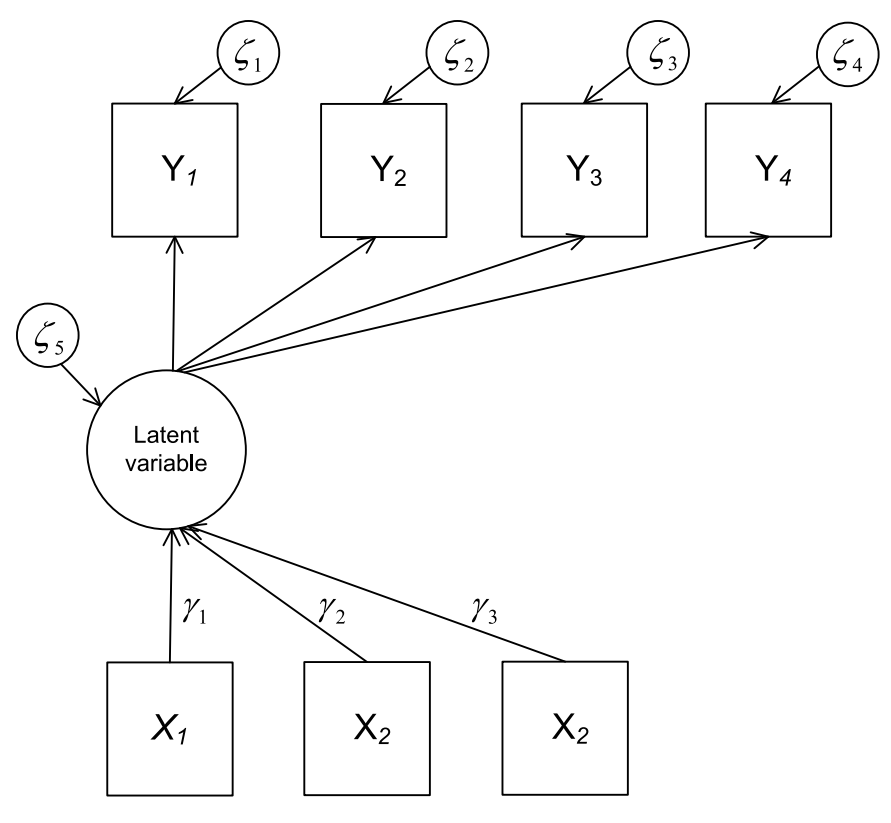

FIGURE 8 Hypothetical SEM model.

So, although inequality constraints can be tested using the approach proposed, further research should focus on decreasing computation time. We therefore recommend implementing our procedure in Mplus. If this can be achieved, the method will be attractive for researchers like Deković et al. (2004), because they will be able to evaluate informative hypotheses easily and quickly.

\section{ACKNOWLEDGMENT}

This work was supported by a grant from the Netherlands Organization for Scientific Research (NWO-VICI-453-05-002).

\section{REFERENCES}

Andrews, D. W. K. (1996). Admissibility of the likelihood ratio test when the parameter space is restricted under the alternative. Econometrica, 64, 705-718.

Andrews, D. W. K. (2000). Inconsistency of the bootstrap when a parameter is on the boundary of the parameter space. Econometrica, 68, 399-405.

Barlow, R. E., Bartholomew, D. J., Bremner, H. M., \& Brunk, H. D. (1972). Statistical inference under order restrictions. New York: Wiley.

Bayarri, M. J., \& Berger, J. O. (2000). P-values for composite null models. Journal of the American Statistical Association, 95, 1127-1142.

Berger, V. W., \& Ivanova, A. (2002). The bias of linear rank tests when testing for stochastic order in ordered categorical data. Journal of Statistical Planning and Inference, 107, 237-247.

Bollen, K. (1989). Structural equation modeling with latent variables. New York: Wiley. 
Chongcharoen, S., Singh, B., \& Wright, F. (2002). Powers of some one-sided multivariate tests with the population covariance matrix known up to a multiplicative constant. Journal of Statistical Planning and Inference, 107, $103-121$.

Deković, M., Noom, M. J., \& Meeus, W. (1997). Expectations regarding development during adolescence: Parental and adolescent perceptions. Journal of Youth and Adolescence, 26, 253-272.

Deković, M., Wissink, I., \& Meijer, A. M. (2004). The role of family and peer relations in adolescent antisocial behaviour: Comparison of four ethnic groups. Journal of Adolescence, 27, 497-514.

Dishion, T. J., \& McMahon, R. J. (1998). Parental monitoring and the prevention of child and adolescent problem behavior: A conceptual and empirical foundation. Clinical Child and Family Psychology Review, 1, 61-75.

Efron, B., \& Tibshirani, R. J. (1993). An introduction to the bootstrap. New York: Chapman \& Hall.

Galindo-Garre, F., \& Vermunt, J. (2004). The order-restricted association model: Two estimation algorithms and issues in testing. Psychometrika, 69, 641-654.

Galindo-Garre, F., \& Vermunt, J. (2005). Testing log-linear models with inequality constraints: A comparison of asymptotic, bootstrap, and posterior predictive p-values. Statistica Neerlandica, 59, 82-94.

Gonzalez, R., \& Griffin, D. (2001). Testing parameters in structural equation modelling: Every "one" matters. Psychological Methods, 6, 258-269.

Greenberger, E., \& Chen, C. (1996). Perceived family relationships and depressed mood in early and late adolescence: A comparison of European and Asian American. Developmental Psychology, 32, 707-716.

Han, S. (1977). A globally convergent method for nonlinear programming. Journal of Optimization Theory and Applications, 22/23, 297-309.

Hoijtink, H., Klugkist, I., \& Boelen, P. A. (Eds.). (2008). Bayesian evaluation of informative hypotheses. New York: Springer.

Khalil, G., Saikali, R., \& Berger, L. (2002). More powerful tests for the sign testing problem. Journal of Statistical Planning and Inference, 107, 187-205.

Klugkist, I., Laudy, O., \& Hoijtink, H. (2005). Inequality constrained analysis of variance: A Bayesian approach. Psychological Methods, 10, 477-493.

Lee, C. C., \& Yan, X. (2002). Chi-squared tests for and against uniform stochastic ordering on multinomial parameters. Journal of Statistical Planning and Inference, 107, 267-280.

Mounts, N. S., \& Steinberg, L. (1995). An ecological analysis of peer influence on adolescent grade point average and drug use. Developmental Psychology, 31, 915-922.

Muthén, L. K., \& Muthén, B. O. (2007). Mplus: Statistical analysis with latent variables: User's guide. Los Angeles, CA: Muthén \& Muthén.

Perlman, M. D., \& Wu, L. (2002a). A class of conditional tests for a multivariate one-sided alternative. Journal of Statistical Planning and Inference, 107, 155-171.

Perlman, M. D., \& Wu, L. (2002b). A defense of the likelihood ratio test for one-sided and order-restricted alternatives. Journal of Statistical Planning and Inference, 107, 173-186.

Phalet, K., \& Schönpflung, U. (2001). Intergenerational transmission of collectivism and achievement values in two acculturation contexts: The case of Turkish families in Germany and Turkish and Moroccan families in the Netherlands. Journal of Cross-Cultural Psychology, 32, 186-201.

Ritov, Y., \& Gilula, Z. (1993). Analysis of contingency tables by correspondence models subject to order constraints. Journal of the American Statistical Association, 88, 1380-1387.

Robertson, T., Wright, F. T., \& Dykstra, R. L. (1988). Order restricted statistical inference. New York: Wiley.

Sampson, A. R., \& Singh, H. (2002). Min and max scorings for two sample partially ordered categorical data. Journal of Statistical Planning and Inference, 107, 219-236.

Schoenberg, S. (1997). Constrained maximum likelihood. Computational Economics, 10, 251-266.

Sen, P. K., \& Silvapulle, M. J. (2002). An appraisal of some aspects of statistical inference under inequality constraints. Journal of Statistical Planning and Inference, 107, 3-43.

Silvapulle, M. J., \& Sen, P. K. (2004). Constrained statistical inference: Order, inequality, and shape constraints. London: Wiley.

Silvapulle, M. J., Silvapulle, P., \& Basawa, I. V. (2002). Tests against inequality constraints in semiparametric models. Journal of Statistical Planning and Inference, 107, 307-320.

Stoel, R. D., Galindo-Garre, F., Dolan, C., \& Van den Wittenboer, G. (2006). On the likelihood ratio test in structural equation modeling when parameters are subject to boundary constraints. Psychological Methods, 4, 439-455.

Tsonaka, R., \& Moustaki, I. (2007). Parameter constraints in generalized linear latent variable models. Computational Statistics and Data Analysis, 51, 4164-4177. 